\title{
A Clustering Based Approach for Automatic Image Segmentation: An Application to Biplane Ventriculograms
}

\author{
Antonio Bravo ${ }^{1}$, Rubén Medina², and J. Arelis Díaz ${ }^{3}$ \\ ${ }^{1}$ Grupo de Bioingeniería, Universidad Nacional Experimental del Táchira, Decanato \\ de Investigación, San Cristóbal 5001, Venezuela \\ abravo@unet.edu.ve \\ ${ }^{2}$ Grupo de Ingeniería Biomédica (GIBULA), Universidad de Los Andes, Facultad de \\ Ingeniería, Mérida 5101, Venezuela \\ ${ }^{3}$ Laboratorio de Investigación en Matemática Pura y Aplicada, Universidad Nacional \\ Experimental del Táchira, Decanato de Investigación, San Cristóbal 5001, Venezuela \\ jdiaz@unet.edu.ve
}

\begin{abstract}
This paper reports on an automatic method for ventricular cavity segmentation in angiographic images. The first step of the method consists in applying a linear regression model that exploits the functional relationship between the original input image and a smoothed version. This intermediate result is used as input to a clustering algorithm, which is based on a region growing technique. The clustering algorithm is a two stage process. In the first stage an initial segmentation is achieved using as input the result of the linear regression and the smoothed version of the input image. The second stage is intended for refining the initial segmentation based on feature vectors including the area, the gray-level average and the centroid of each candidate region. The segmentation method is conceptually simple and provides an accurate contour detection for the left ventricle cavity.
\end{abstract}

\section{Introduction}

Clustering is the division of a dataset into groups of similar objects. Clustering methods have been used for recognition of shapes [1] [2]. Given a set of $M$ data points, the objective is to detect similarities between points of this data set. The clustered data is stored in $d$-dimension vectors. Each vector represents a particular object which is described by $d$ features that enables comparison to other data vectors. The result of the feature comparison process is used to classify the data into several disjoint subsets. This technique has been used to solve the image segmentation problem. Clustering based segmentation considers features like pixel space positions, topological relations and contour features. Examples of these techniques are the methods for classification [3] and clustering by Region Growing [4].

Region growing methods have been used for performing the segmentation of several medical imaging modalities [5]. The region growing is usually based on 
simple linkage, on multiple connections or centroid based linkage [6]. An alternative classification method is based on clustering by graph theory [7]. According to this method, data is initially represented by adjacent graphs 8 building up several subgraph sets whose union represents a spatially connected region in the image. The fuzzy $\mathrm{C}-$ means classification algorithms have also provided good results for image segmentation. These methods require a high computational cost [9] 10]. Ventriculograms are obtained from a medical imaging modality based on X-rays, after the injection of a contrast medium in the cavities of the heart aiming to enhance the contrast with respect to other tissues. Such examination enables the assessment of morphology and function of the heart. Ventriculographic image analysis requires a precise description of ventricular shape in order to quantify the parameters associated with the cardiovascular function [11] 12] or alternatively for performing the visualization of this anatomical structure [13]. The accurate description of ventricular shape and their quantitative analysis is important, since cardiovascular disease (CVD) accounts for one third of the deaths in the world [14.

Recently, several robust methods for ventriculographic image segmentation have been proposed. Suzuki et al. 15] have developed a ventricular contour detector based on neural networks (NN). The detector was implemented using a multilayer neural network which was trained through a back-propagation algorithm. The training set includes left ventricle images and ventricular contours traced by a cardiologist. Validation was performed by comparison of the area enclosed by the estimated contour with respect to the reference contour traced by the cardiologist. The average contour error obtained at end-diastole was $6.2 \%$. Oost et al. 16 have proposed a ventricular cavity automatic segmentation method based on Active Appearance Models (AAMs) and dynamic programming (DP). The active appearance model is used to exploit the existing correlations in shape and texture between end-diastole and end-systole images. A dynamic programming algorithm was used to incorporate cardiac motion features to the method. The method was evaluated by using 140 images. The average border positioning error was smaller than $1.45 \mathrm{~mm}$. These methods provided an accurate representation of ventricular borders, however, they are not yet fully validated and accepted in the clinical work as a gold standard.

The objective of this research is to develop a left ventricle segmentation method based on region growing. This segmentation method consists of two fundamental stages. The first is a preprocessing stage that enhances the image data using statistical tools. The second is a classification stage based on region growing. The method uses a feature vector including the gray-level intensity and the average of pixels included in a neighborhood.

\section{Method}

\subsection{Preprocessing Stage}

An average filter is applied to the input image. According to this filter, if a pixel value is greater than the average of its neighbors (the eight closest pixels in a 
neighborhood of size $3 \times 3$ ) plus a certain threshold $\varepsilon$, then the pixel value in the output image is set to the average value, otherwise the output pixel is set equal to the pixel in the input image. The output image is a smoothed version of the input. The threshold value $\varepsilon$ was set to the standard deviation of the input image.

A linear regression model is estimated to exploit the functional relation [17] between the data of the input image $\left(\mathbf{I}_{O}\right)$ and the data of the smoothed image $\left(\mathbf{I}_{P}\right)$. The model is described by:

$$
\hat{\mathbf{I}}_{P}=\beta_{0}+\beta_{1} \mathbf{I}_{O},
$$

where $\beta_{0}$ and $\beta_{1}$ are the regression coefficients [17, which are estimated by the least squares method according to:

$$
\begin{aligned}
& \beta_{0}=\frac{\sum_{i=0}^{n}\left(I_{O_{i}}-\overline{\mathbf{I}}_{O}\right)\left(I_{P_{i}}-\overline{\mathbf{I}}_{P}\right)}{\sum_{i=0}^{n}\left(I_{O_{i}}-\overline{\mathbf{I}}_{O}\right)^{2}} \\
& \beta_{1}=\overline{\mathbf{I}}_{P}-\beta_{0} \overline{\mathbf{I}}_{O}
\end{aligned}
$$

The data obtained by the functional relation between the original image and the smoothed image represents a new image $\left(\hat{\mathbf{I}}_{P}\right)$. The pixel values in this image and in the smoothed image are the members of the feature vector used in the clustering method.

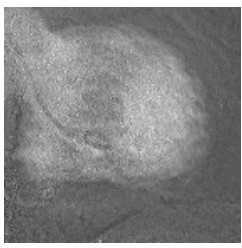

(a)

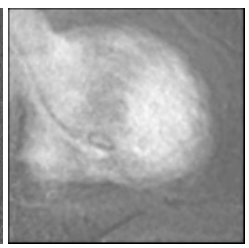

(b)

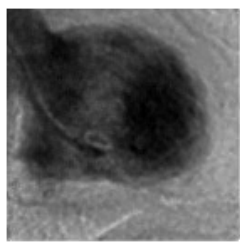

(c)

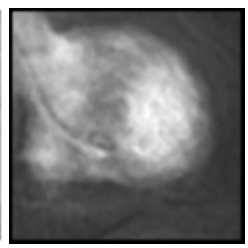

(d)

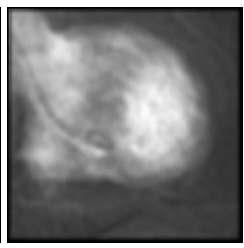

(e)

Fig. 1. Linear regression model. (a) Original image. (b) Smoothed image. (c) Linear regression image. (d) Similarity image. (e) Density function image.

\subsection{Clustering Stage}

The clustering stage is a four steps process. In the first step a similarity matrix is generated based on a similarity criterion [6] that measures the difference between the gray-level values of pixels in $\hat{\mathbf{I}}_{P}$ and the smoothed image $\left(\mathbf{I}_{P}\right)$. According to this criterion, pixels $p_{1}(u, v)$ (in $\hat{\mathbf{I}}_{P}$ ) and $p_{2}(u, v)$ (in $\mathbf{I}_{P}$ ) have feature vectors denoted as: $\mathbf{p} \mathbf{v}_{1}=\left[i_{1}, a\right]$ and $\mathbf{p v}_{2}=\left[i_{2}, b\right]$, where $i_{1}$ and $i_{2}$ denote the intensities associated with the corresponding pixel and, $a$ and $b$ are the intensity average in a $5 \times 5$ neighborhood around each pixel. The neighborhood size was varied between $3 \times 3$ and $11 \times 11$. The size $5 \times 5$ provided the best results and was retained. In general the impact of neighborhood size is not very important; 


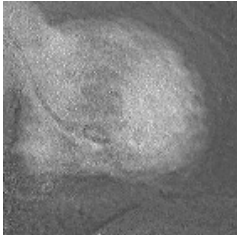

(a)

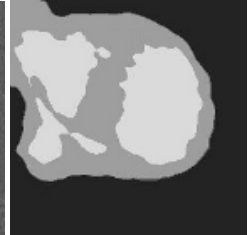

(b)

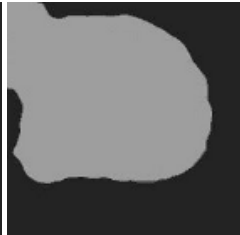

(c)

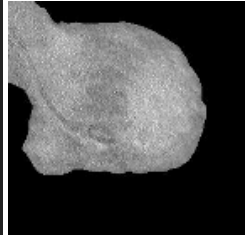

(d)

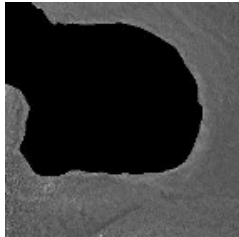

(e)

Fig. 2. (a) Original image (RAO view). (b) Approximate segmentation. (c) Final clustering. (d) Left ventricle cavity. (e) Background region.

however, neighborhood sizes larger than $5 \times 5$ increase the computational cost. The similarity matrix $\left(\mathbf{I}_{S}\right)$ is an image obtained using the following equation:

$$
\mathbf{I}_{S}=\left(i_{1}-i_{2}\right)^{2}+\left(i_{1}-b\right)^{2}+\left(i_{2}-a\right)^{2} .
$$

In the second step a data density function $\mathbf{I}_{D}$ [18] is obtained by convolving $\mathbf{I}_{S}$ with a unimodal density mask $\mathbf{K}$ as follows:

$$
\mathbf{I}_{D}=\mathbf{I}_{S} * \mathbf{K}
$$

The density function establishes the degree of dispersion in $\mathbf{I}_{S}$. A Gaussian distribution with standard deviation $\sigma$ is used as a density mask [18] as follows:

$$
K(i, j)=\left(\frac{1}{2 \pi \sigma^{2}}\right)^{n / 2} e^{\frac{-|i+j|^{2}}{2 \sigma^{2}}} ; 0 \leq i, j \leq n,
$$

where $n$ denotes the mask size. In our case we use $n=5$ and $\sigma$ is set as the standard deviation of the smoothed image $\mathbf{I}_{P}$.

In the third step, an approximate segmentation is performed. All pixels that have an intensity value lower than the standard deviation of image $\mathbf{I}_{P}$ are considered as seed points for a simple linkage region growing algorithm that is performed on image $\mathbf{I}_{D}$. A region grows from each of the seed pixels by grouping neighbor pixels that comply with the following uniformity criterion: if the difference between two neighbor pixels is lower than the standard deviation $\sigma_{D}$ of image $\mathbf{I}_{D}$, then both pixels are clustered. This process is applied to the entire image $\mathbf{I}_{D}$ until all pixels are clustered and tagged. Thus, results achieved provide an approximate segmentation of the input image as more than two regions can be obtained.

The last step of the clustering process improves the segmentation by using a region growing method by multiple linkage [19. The objective is to obtain only two regions partitioning the ventriculograms: one representing the interior of the left ventricle cavity and the other the background region. Each tagged region obtained in the previous step is a seed region that is represented by a feature vector whose components are the area, the average intensity and the centroid. Neighbor regions are merged when the following uniformity criterion is met: the neighbor regions must provide a minimum for the difference between 
the average intensity, for the difference between the area and for the Euclidean distance between centroids. This process is applied in an iterative way until only two neighbor regions are left.

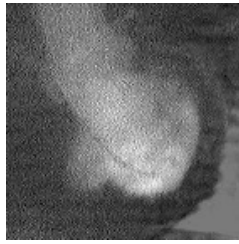

(a)

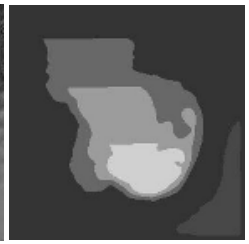

(b)

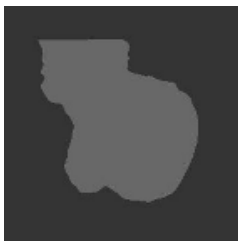

(c)

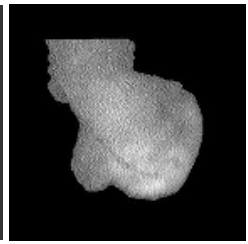

(d)

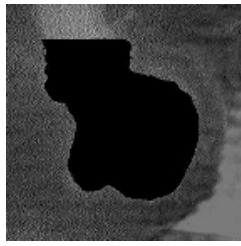

(e)

Fig. 3. (a) End-systole image (LAO view). (b) Approximate segmentation. (c) Clustering results. (d) Left ventricle cavity. (e) Background region.

\section{Results}

The proposed method has been tested with biplane ventriculograms acquired at several instants of the cardiac cycle. The biplane images were acquired according

Table 1. Components of the feature vector for each of the regions in the end-diastole image

\begin{tabular}{c||c|c|c}
\hline Feature vector & Area (pixels) & Gray-level average & Centroid \\
\hline \hline Region 0 & 13305 & 216 & $(120,98)$ \\
\hline Region 1 & 16995 & 152 & $(105,106)$ \\
\hline Region 2 & 35236 & 31 & $(145,155)$ \\
\hline \hline
\end{tabular}

Table 2. Feature vector for each of the regions in the end-systole image

\begin{tabular}{c||c|c|c}
\hline Feature vector & Area (pixels) & Gray-level average & Centroid \\
\hline \hline Region 0 & 35421 & 52 & $(62,67)$ \\
\hline Region 1 & 4515 & 72 & $(236,220)$ \\
\hline Region 2 & 15172 & 104 & $(103,81)$ \\
\hline Region 3 & 7111 & 156 & $(135,116)$ \\
\hline Region 4 & 3317 & 208 & $(144,174)$ \\
\hline \hline
\end{tabular}

Table 3. Feature vector for each of the regions after the first iteration of the multiple linkage clustering in the end-systole image

\begin{tabular}{c||c|c|c}
\hline Feature vector & Area (pixels) & Gray-level average & Centroid \\
\hline \hline Region 0 & 39936 & 52 & $(62,67)$ \\
\hline Region 1 & 15172 & 104 & $(103,81)$ \\
\hline Region 2 & 10428 & 182 & $(132,144)$ \\
\hline \hline
\end{tabular}




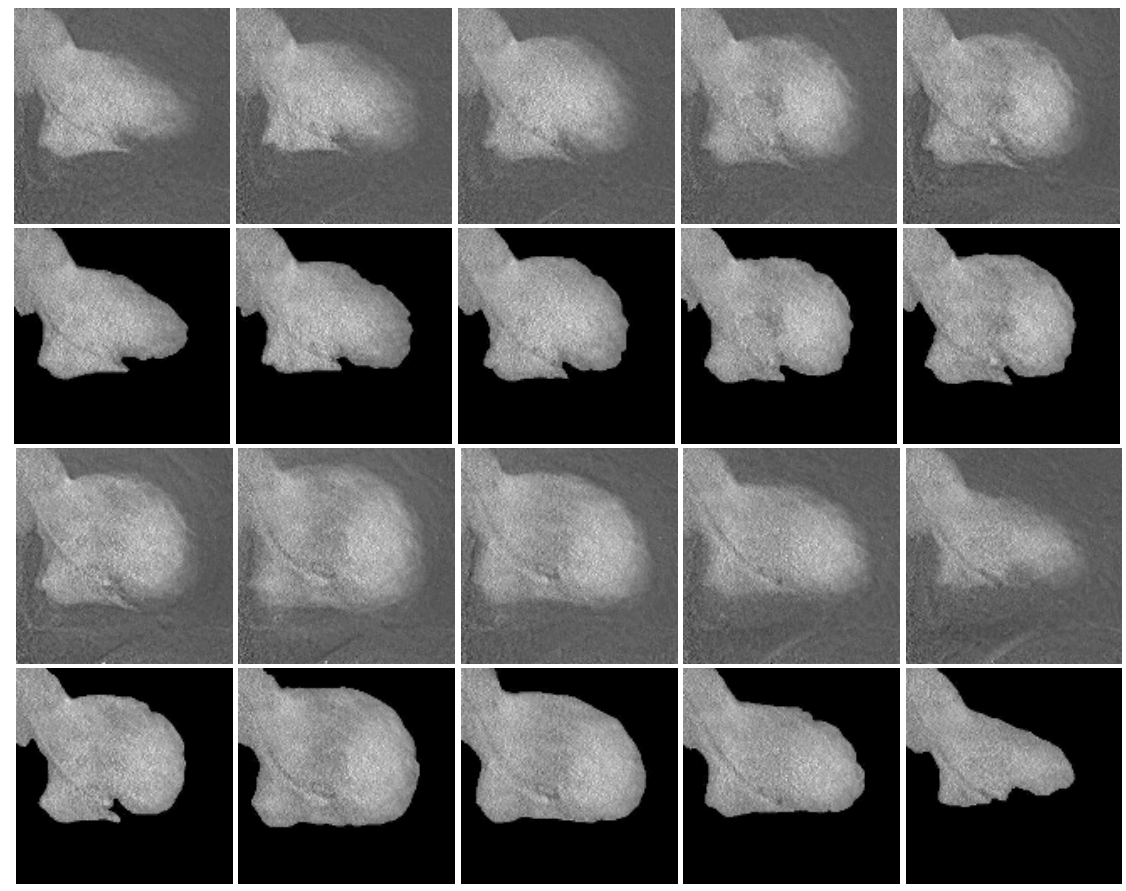

Fig. 4. Segmentation for a RAO view image sequence

to the conventional Left Anterior Oblique (LAO) $60^{\circ}$ and Right Anterior Oblique (RAO) $30^{\circ}$ views. All images are of size $256 \times 256$ pixels. The input images were preprocessed by performing distortion correction and logarithmic subtraction.

Figure 11a shows the input image that corresponds to the RAO view at the end-diastole instant. Figure 1,b shows the smoothed image using a neighborhood of size $3 \times 3$ and figure 11.c shows the image estimated by linear-regression $\left(\hat{\mathbf{I}}_{P}\right)$. The similarity image (obtained using equation 3) is shown in figure 1. d. This image is obtained as a quadratic function that combines pixels in the original image with pixels in the smoothed image. As a result the output image has a smoothed appearance. Finally, the density function image is shown in figure 1. This image is obtained by convolving a Gaussian function with the similarity image, thus increasing the degree of smoothing. The standard deviation value $(\sigma=44.63)$ is estimated from the smoothed image $I_{P}$.

Figure 2]b shows the approximate segmentation where several regions are identified. The standard deviation used for determining the 3 clusters was $\sigma_{D}=$ 59.63. This standard deviation is estimated from the density function image $I_{D}$. A feature vector for each of the regions is shown in Table 1. The adjacent regions with a more similar feature vector are regions 0 and 1 . The clustering step using multiple linkage provides the final segmentation shown in figure 2.c, where regions 0 and 1 are clustered based on the region growing step. Figure 2 , d2. e shows the two regions obtained from the segmentation process. In figure 3 , 


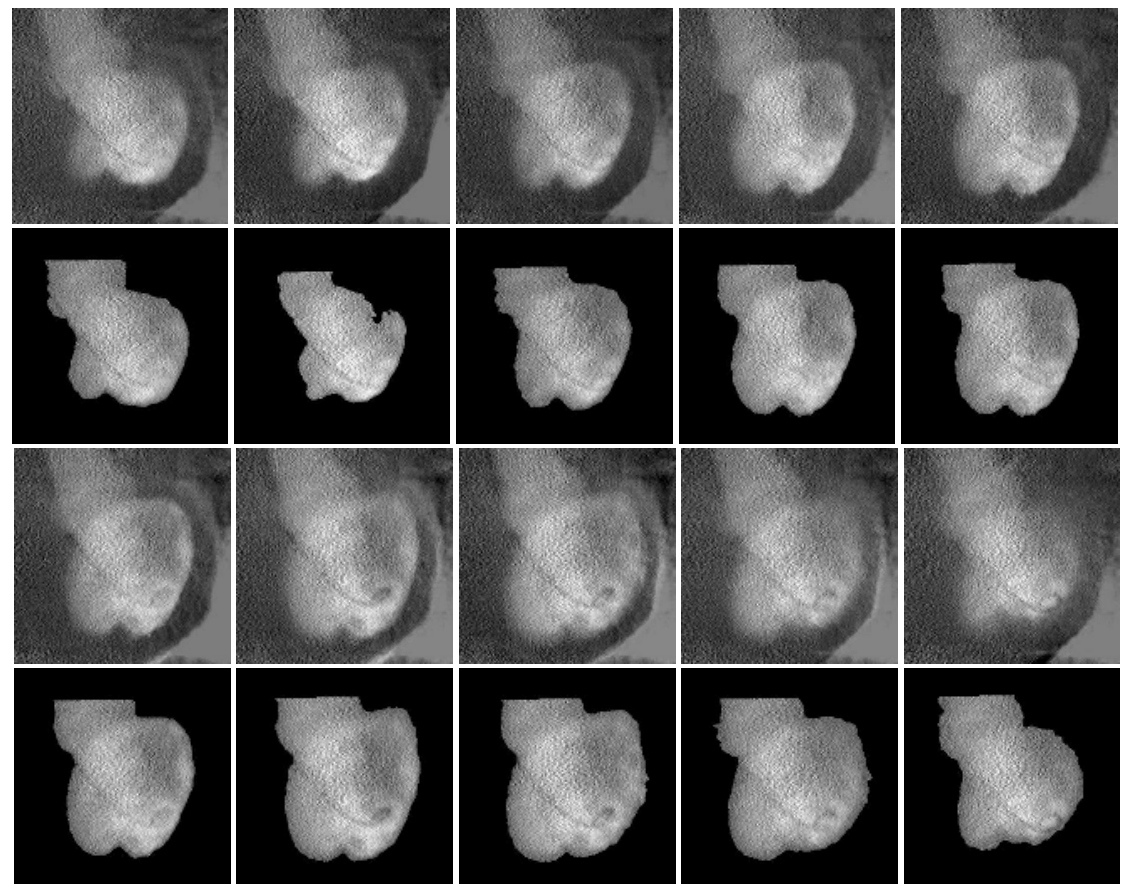

Fig. 5. Segmentation for a LAO view image sequence

results of the segmentation for the end-systole ventriculogram (LAO view) are shown. In this case, five regions have been found at the approximate segmentation step. Table 2 shows the feature vectors for each region. The clustering step is performed using two iterations for attaining the final segmentation. During the first iteration regions 0 and 1 are merged as well as regions 3 and 4 . The merged regions have the feature vectors shown in Table 3. During the second iteration regions 1 and 2 are merged providing the final segmentation shown in figure 3. .

Figure 4 and Figure 5 show the results of the segmentation for a sequence of ventriculograms in the RAO view and LAO view respectively. The first and third rows (from top to bottom) show the original images while the second and fourth rows show the segmentation results.

Validation of the segmentation method is performed by quantifying the difference between the left ventricle shape obtained with respect to the left ventricle shape traced by a cardiologist. The error is expressed as the ratio between the area of the shape difference with respect to area of the union of shapes compared. The error obtained (mean \pm standard deviation) for a sequence of ventriculograms in the RAO view, including 25 images is $5.47 \% \pm 1.61 \%$, with a maximum value of $7.84 \%$ and a minimum value of $2.53 \%$. When the segmentation is performed for the sequence showing the LAO view (including 25 images) the error is $4.39 \% \pm 3.41 \%$ with a maximum value of $13.90 \%$ and a minimum value of 


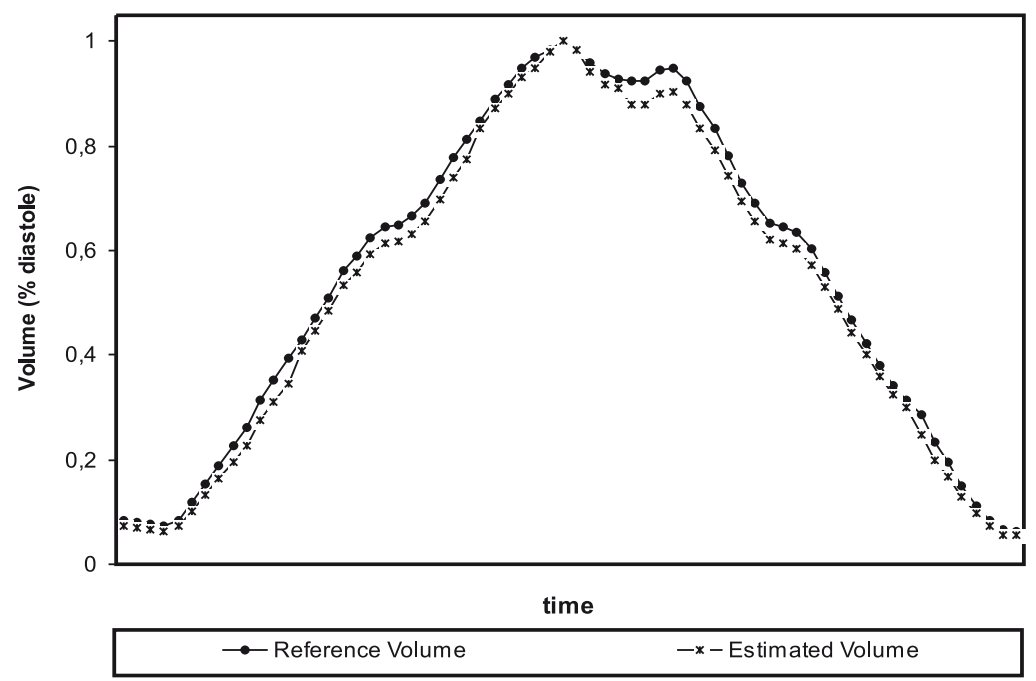

Fig. 6. Left ventricle volume

$1.53 \%$. The error considering the set of 50 images (25 for the RAO view and 25 for the LAO view) is $4.93 \% \pm 2.69 \%$. The volume for the left ventricle during the cardiac cycle is estimated using the Area-Length method 20] from the contours obtained using the segmentation method. Fig. 6] shows the left ventricle volume estimated using the contours obtained by the proposed segmentation method (estimated volume) and the volume obtained from contours traced by the cardiologist (reference volume). The volume is normalized with respect to the end-diastole volume. The error obtained by comparing the reference volume with respect to the estimated volume is $4.61 \% \pm 1.22 \%$. The ejection fraction 21] for the reference volume is 0.77 and 0.74 for the estimated volume. The percent error is $3.9 \%$.

\section{Conclusions}

An automatic image segmentation method has been presented. The proposed method is tested using real biplane ventriculograms. The region growing algorithm used as the core of the segmentation process enables the accurate discrimination of the ventricular cavity. The segmentation method does not require any prior knowledge about the ventriculograms and considers the relationship between neighbor pixels.

The segmentation method is conceptually simple and provides an accurate left ventricle contour. The method requires a low computational cost. The proposed approach provides an initial segmentation that is later improved using the multiple linkage region growing algorithm. The initial segmentation could 
be interpreted as a compression stage that codes the set of groups that represent the image. The initial segmentation could also be considered as the input to other segmentation methods based on clustering or non-supervised learning machines (for instance auto-organized maps or fuzzy logic based techniques). The tests performed on real biplane ventriculograms shows that parameters describing the cardiac function like the volume and the ejection fraction are consistent with the values reported in the literature [22] 23].

As a future research we propose to incorporate other components to the feature vector such as contour curvature and smoothness. In addition, comparison to other methods would be performed. A complete validation is also necessary, including an important number of control subjects as well as cardiac patients. In the validation stage we plan to use other metrics for comparing the segmentation results as for instance the average contour positioning error as in [16. The validation stage could also include a comparison of estimated parameters describing the cardiac function with respect to results obtained using other imaging modalities like magnetic resonance imaging or multi-slice computerized tomography.

\section{Acknowledgment}

The authors would like to thank the Investigation Dean's Office of Universidad Nacional Experimental del Táchira (San Cristóbal, Venezuela) and CDCHT from Universidad de Los Andes (Mérida, Venezuela) for their support to this project.

\section{References}

1. R. Duda, P. Hart, and D. Stork. Pattern Recognition and Scene Analysis (2nd Edition). John Wiley and Sons, New York, 2000.

2. J. Hubert. Some applications of graph theory to clustering. Psychometrika, 39(3):283-309, 1974.

3. R. Horaud and O. Monga. Vision par ordinateur, outils fondamentaux. HERMES, Paris, 1993.

4. M. Sonka, V. Hlavac, and R. Boyle. Image Processing, Analysis and Machine Vision. PWS Publishing, Pacific Grove, 1999.

5. T. M. Lehmann, D. Beier, C. Thies, and T. Seidl. Segmentation of medical images combining local, regional, global, and hierarchical distances into a bottom-up region merging scheme. In Proceedings of SPIE, volume 5747, pages 546-555, 2005.

6. R. Haralick and L. Shapiro. Computer and Robot Vision, volume I. Addison-Wesley Publishing Company, USA, 1992.

7. Z. Wu and R. Leahy. An optimal graph theoretic approach to data clustering: Theory and its application to image segmentation. IEEE Trans. Pattern Recognition Analysis and Machine Inteligence, 15(11):1101-1113, 1993.

8. R. Urquhart. Graph theoretical clustering based on limited neighborhood sets. Pattern Recognition, 13(3):173-187, 1982.

9. R. Medina, M. Garreau, D. Jugo, C. Castillo, and J. Toro. Segmentation of ventricular angiographic images using fuzzy clustering. In Proceedings of the 17th Annual International Conference of the IEEE EMBS, pages 405-406, Montreal, 1998. 
10. J. Richardt, F. Karl, C. Müller, and R. Klette. The fuzzy local-global duality in detecting pictorial pattern. Pattern Recognition Letters, 17(2):187-195, 1996.

11. J. Kennedy, S. Trenholme, I. Kaiser, and S. Wash. Left ventricular volume and mass from single-plane cineangiocardiogram. A comparison of anteroposterior and right anterior oblique methods. American Heart Journal, 80(3):343-352, 1970.

12. Osman Ratib. Quantitative analysis of cardiac function. In Isaac Bankman, editor, Handbook of Medical Imaging: Processing and Analysis, pages 359-374. Academic Press, San Diego, 2000.

13. R. Medina, M. Garreau, J. Toro, J. L. Coatrieux, and D. Jugo. Three-dimensional reconstruction of left ventricle from two angiographic views: An evidence combination approach. IEEE Transactions on Systems, Man, and Cybernetics-Part A: Systems and Humans, 34(3):359-370, 2004.

14. Reducing risk and promoting healthy life. The World Health Report 2002, Geneva, World Health Organization, Julio 2002.

15. K. Suzuki, I. Horiba, N. Sugie, and M. Nanki. Extraction of left ventricular contours from left ventriculograms by means of a neural edge detector. IEEE Transactions on Medical Imaging, 23(3):330-339, 2004.

16. E. Oost, G. Koning, M. Sonka, J. Reiber, and B. Lelieveldt. Automated segmentation of $\mathrm{X}$-ray left ventricular angiograms using multi-view active appearance models and dynamic programming. In Proceedings of the Functional Imaging and Modeling of the Heart: Third International Workshop, pages 23-31, Barcelona, Spain, 2005.

17. J. Neter, W. Wasserman, and G. Whitmore. Applied Statistics. Allyn and Bacon, Boston, 1992.

18. E. Pauwels and G. Frederix. Finding salient regions in images: Non-parametric clustering for images segmentation and grouping. Computer Vision and Image Understanding, 75(1,2):73-85, 1999. Special Issue.

19. R. Haralick and L. Shapiro. Survey: Image segmentation techniques. Computer Vision Graphics and Image Processing, 29(1):100-132, 1985.

20. H. Dodge, H. Sandler, D. Ballew, and J. Lord. The use of biplane angiocardiography for the measurement of the left ventricular volume in man. American Heart Journal, 60:762-776, 1960.

21. S. Yan, B. Lamberto, M. Vladir, and G. Harry. From Cardiac Catheterization Data to hemodynamic Parameters. F. A. Davis Company, USA, 1978.

22. J. Kennedy, W. Baxley, M. Figley, H. Dodge, and J. Blackmon. Quantitative angiocardiography. I. The normal left ventricle in man. Circulation, 34(2):272$278,1966$.

23. K. Hammermeister, R. Brooks, and J. Warbasse. Rate of change of left ventricular volume in man. I. Validation of peak systolic ejection rate in health and disease. Circulation, 49(4):729-738, 1974. 